\title{
Las TIC y los procesos de envejecimiento activo: ¿una promesa incumplida?
}

\section{ICTs and active aging processes: a broken promise?}

\author{
Mayte Muñoz Alarcón”, Juan Sáez Carreras**, Margarita Campillo Díaz***
}

Recibido: 13 de marzo de 2020 Aceptado: 6 de abril de 2020 Publicado: 31 de julio de 2020

To cite this article: Muñoz, M., Sáez, J., Campillo, M. (2020). Las TIC y los procesos de envejecimiento activo: ¿una promesa incumplida? Márgenes, Revista de Educación de la Universidad de Málaga, 1 (2), 34-47

DOI: http://dx.doi.org/10.24310/mgnmar.v1i2.8287

\section{RESUMEN}

El impacto de las nuevas tecnologías de la información y la comunicación -TIC- está por medir y por definir con precisión. Su influjo, no cabe duda, es progresivamente mayor en contextos crecientes en redes telemáticas y un tejido social cada vez más mediatizado. Entre el optimismo y el pesimismo de los discursos relacionados con estas herramientas son escasos las reflexiones de fondo, así como los estudios sólidos que nos permitan conocer su emergencia, aplicación, desarrollo y resultados. En el territorio de las personas mayores, ya sea por analogismo con otras edades o ya por extensión del potencial que encierran, entre otras razones, van en aumento las llamadas a que sean utilizadas con más frecuencia las TIC en los procesos de envejecimiento en que ellas se ven envueltas, tanto a nivel personal como social. Este trabajo tiene por meta explorar las relaciones que existen o pueden existir entre las personas mayores y las TIC a partir del lema lanzado, en 2002, sobre “envejecimiento activo". Una noción o expresión vinculada en Europa y América a promesas de carácter político, social, económico y cultural y, por tanto, impulsora de la mejora de la calidad de vida de las personas que envejecen en sociedades, avanzadas y en desarrollo, cuyos cambios y transformaciones multiplican sus miedos, riesgos de exclusión e inseguridades. Nuestra exploración de corte analítico y apoyo empírico, trata de investigar si tal promesa se cumple objetivamente o si, por el contrario, forma parte de la retórica al uso o de los discursos políticos de carácter populista y electoralista.

Palabras clave: envejecimiento activo; personas mayores; nuevas tecnologías de la información y la comunicación; formación; políticas sociales y educativas

\section{ABSTRACT}

The impact of the new information and communication technologies (ICTs) has yet to be measured and accurately defined. They are undoubtedly having increasing impact in growing areas of telematic networks and an increasingly controlled social network. Between the optimism and pessimism of the discourse related to these tools, there has been scanty in-depth consideration or solid studies to enable us to understand how they emerge, are applied and, developed or the outcomes. In the context of the elderly, either by analogy with other age groups or for their potential, among other reasons, there are growing calls for more frequent use of ICTs in people's personal and social ageing processes. This work seeks to explore the relationships that exist or could exist between the elderly and ICTs based on the slogan launched in 2002 on "active ageing", a notion or expression linked in Europe and America to promises of a political, social, economic and cultural nature and

*Mayte Muñoz Alarcón 0000-0003-4463-1843

Departamento Teoría e Historia de la Educación, Universidad de Murcia (España)

mteresa.munoz@um.es
*Juan Sáez Carreras 0000-0002-0043-9254

Catedrático Dpto. Teoría e Historia de la Educación, Universidad de Murcia.
*** Margarita Campillo Díaz 0000-0003-4463-1843 Profesora Dpto. Teoría e Historia de la Educación, Universidad de Murcia. 


\section{ESTUDIOS Y E N SAYOS}

therefore the promoter of improved quality of life for people who grow old in advanced and developing societies that change and transform in ways that multiply their fears, risk of exclusion and insecurities. Nour exploration of analytical and empirical support tries to verify if such a promise is objectively fulfilled or whether, on the contrary, it forms part of the rhetoric of the use or political discourses of a populist and electoralist nature. empirical, tries to verify if such a promise is fulfilled objectively or if, on the contrary, it forms part of the rhetoric to the use or of the political speeches of populist and electoralist character.

Keywords: active ageing; the elderly; new information and communication technologies; trainin; social and educational policies

\section{INTRODUCCIÓN}

La pregunta central que dirige este trabajo es: ¿qué usos de las Tecnologías de la Información y la Comunicación (TIC) hacen las personas mayores inmersas en sus respectivos procesos de envejecimiento? y, ¿en qué modo contribuyen a mejorar sus necesidades y expectativas vitales? Tratando de ir más allá de las cuestiones tópicas que, en el caso de las TIC se repiten en la mayoría de los discursos, algunas otras interrogaciones asociadas surgen al hilo de formular la primera, tales como ¿hasta qué punto tienen los mayores necesidad de aprender las nuevas tecnologías?, ¿cuáles son los efectos de este uso?, ¿qué estudios se han llevado a cabo tratando de explorar las relaciones entre las nuevas tecnologías y su utilización por las personas mayores?, ¿qué papel cumple la formación cuando se la relaciona con las personas mayores y las nuevas tecnologías? El presente estudio trata de responder básicamente a la primera pregunta, aunque no reniega a abordar, tangencialmente, algunas de las que hemos vinculado a la cuestión central. De ahí que, como corresponde a todo trabajo canónico analítico, y deseando ir más allá de las respuestas convencionales al uso, nuestra tarea se articule en torno a tres focos de estudio. Abordaremos, en primer lugar, el significante "envejecimiento activo", expresión recurrente en nombre de la que, esencialmente en Europa y América, se están llevando a cabo numerosos proyectos de investigación, así como múltiples actividades de carácter político, social, educativo, cultural y económico relacionadas con las personas mayores en su devenir existencial. En un segundo momento nos adentraremos en el binomio que forman las TIC y los mayores deteniéndonos, con más precisión, en los diferentes usos que hacen de ellas y, esencialmente, en las relacionadas con la comunicación y la formación. En este recorrido deseamos preguntarnos, finalmente, por las promesas cumplidas y por aquellas que, a modo de retos, quedan por cumplir en tiempos en los que la brecha digital va disminuyendo gracias a los esfuerzos políticos, económicos y pedagógicos de alfabetización digital. Se trata, en definitiva, de una interpelación a las promesas sostenidas sobre las TIC con deseos reales de escrutar su potencial en el presente y futuro del envejecimiento humano.

\section{2. ¿QUÉ ES LO QUE AÑADE EL ADJETIVO ACTIVO AL SUSTANTIVO ENVEJECIMIENTO?}

Entre el 28 y el 30 de julio de 2010 se celebró en El Escorial un evento, auspiciado por el Ministerio de Sanidad y Política Social (IMSERSO) y la Universidad Complutense de Madrid, en el que colaboraron expertos de diferentes campos relacionados con la vejez. El expresivo título del evento era "Las promesas del envejecimiento activo: investigación, desarrollo e innovación en Europa”, y su finalidad, llevar a cabo una valoración de los objetivos logrados por las políticas y programas impulsados por Europa al amparo del llamado “envejecimiento activo” (OMS, 2002). 


\section{ESTUDIOS Y E N SAYOS}

El envejecimiento activo se presentaba como una plataforma desde la que poder contribuir a mejorar la calidad de vida de las personas y desde la que propiciar un crecimiento sostenido de nuestras sociedades del bienestar. Auspiciando, así, la concreción de políticas y prácticas de diversa índole que aumentaran las oportunidades de envejecer de modo activo a lo largo de la vida.

Ahora bien, las políticas y programas formulados alrededor del envejecimiento activo son muy variables y atienden a distintas áreas del ser humano, lo que dificulta la tarea de articular unos criterios compartidos de evaluación de lo conseguido (Benjamin y Estes, 2003; FernándezBallesteros, 2009). Este hecho se hace más evidente si se piensa en la quiebra o "separación creciente entre el discurso institucional de la Unión Europea y las posiciones sostenidasexpresivamente en los entornos locales-alrededor del envejecimiento activo" (Sánchez y Ferreiro, 2010, p. 5). ¿Qué es lo que está ocurriendo? Si la filosofía de este lema es envejecer mejor a través del cuidado de la salud, la participación social en acciones que redunden en el propio beneficio de los mayores y en el de las personas relacionadas con ellos. Además de la ampliación de los canales de seguridad deseables en tiempos de declives y aumento de la vulnerabilidad y fragilidades, extraña que se traduzca el envejecer activo, tal y como la Comisión Europea lo entiende, por alargar el período de vida de los ciudadanos para mantenerlos "más tiempo vinculados al mundo laboral” (Ídem, p. 8). Esta institucionalización, atravesando la puesta en marcha del lema de la OMS, no solo está evitando que buena parte de la población desee educarse para lograr un verdadero cambio cultural en las creencias, prácticas y estrategias acerca de cómo envejecer mejor, sino también que se reunifiquen posiciones ya caducas como que el envejecimiento es cuestión de edad (¿por qué el modelo no defiende que el envejecimiento es un proceso que se va construyendo desde la infancia y no comienza a partir de los 60 o 65 años?) cuando no se trata de durar más -aunque pueda ser una meta de las personas- sino, sobre todo, durar con calidad vital (Sáez, 2010).

¿Qué es lo que significa, pues, envejecer de modo activo?, frente a otras expresiones dominantes en los discursos sobre los distintos tipos de envejecimiento (productivo, satisfactorio, saludable...) (Caro y Sánchez, 2005), ¿qué es lo que justifica la posición del envejecimiento activo y qué es lo que añade el adjetivo activo al sustantivo envejecimiento para que puedan legitimarse razonablemente las políticas y proyectos que se están impulsando en favor de los mayores? Veamos, en síntesis, muy apretadas, la contribución de las principales ciencias a la clarificación del sustantivo envejecimiento y del adjetivo activo.

\subsection{El aporte de las ciencias: pensando el envejecimiento activo de otra manera}

La filósofa Hanna Arendt promovió un paso clave en la comprensión de la acción humana. En La condición humana (1993, p.199 y ss.) desarrolla una especie de fenomenología de la vida activa al considerar que la naturaleza humana se pluraliza en un "juego de condiciones" que crean o marcan tres tipos de actividad interconectadas y propias del ser humano: el trabajo, la labor y la acción. La labor tiene que ver con la actividad que todo ser humano despliega para "producir todo lo que necesita" como organismo vivo que combate contra la muerte. Es un tipo de actividad "que abarca toda la existencia humana" orientada por intereses biológicos del cuerpo. Y es que "todo lo que nace tiene el deseo de durar". Si la labor es una actividad guiada por un objetivo 


\section{ESTUDIOS Y E N SAYOS}

interno al propio sujeto, actividad de subsistencia, el trabajo se mueve por un objetivo externo al agente, tiene que ver con una dimensión no natural del hombre: es una actividad dedicada a producir artificios y bienes de uso que satisfagan deseos y necesidades. El trabajo convierte al animal humano en "homo faber". Una y otra actividad, labor y trabajo, se pueden realizar pública y aisladamente. La acción, contrariamente, es una actividad eminentemente relacional, no se puede desarrollar en aislado ya que se lleva a cabo a través de la palabra y el discurso lanzados a otros. Nacemos, dice Arendt (1993), en "una trama de relaciones” y la acción se inscribe en el tiempo hasta ser una de las actividades humanas específicas de la "vita activa". La conclusión se desprende sin esfuerzo: si la acción es un testimonio de la existencia humana, vivir es actuar y actuar es vivir. Esto es, la persona que envejece, por el hecho de vivir en relación, de estar inscrita en el tiempo, es activa. Lo es ontológicamente (es decir, a cualquier edad) y empíricamente (ya que la acción se materializa en la actualización de la propia experiencia).

El planteamiento arendtiano nos lleva a explorar algunas de las reflexiones abiertas con anterioridad: ¿acaso los adjetivos añaden algo al sustantivo envejecimiento, siendo la actividad su naturaleza esencial?, ¿no resulta ser tautológica la expresión envejecimiento activo?, ¿hay algún tipo de envejecimiento que nos lleve a concluir que una persona no es activa?, ¿por qué la oposición activo/pasivo, o la de activo con otros adjetivos como saludable, productivo, satisfactorio? Aún más, la cuestión de cuándo deja el mayor de ser activo remite a la necesidad de profundizar sobre las patologías y discapacidades como procesos que pueden impedir el aprendizaje y adelantar tanto la enfermedad como el final de la vida. El espíritu del envejecimiento activo tendría, en este sentido, como uno de sus objetivos posponer la dependencia y la morbilidad y hacerlo en las mejores condiciones vitales. Los recursos que las personas utilicen para lograrlo -entre ellos la educación y la diversidad de artefactos (recuérdese el hombre como artifex de Arnold Gehlen)-son comprensibles y describen el actuar humano. La tecnología no es, por tanto, buena o mala sino lo es en relación a los usos que se hace de ella.

Entre los varios enfoques sociológicos sobre el envejecimiento (teoría de la desvinculación, de la gerotranscendencia, del etiquetamiento social y del intercambio social, entre otros...) se encuentra la teoría de la actividad. Dicha teoría sostiene la necesidad de involucrar a los mayores en actividades sociales. De no hacerlo impulsa una anomia que afecta a la posibilidad misma de darse metas y de trabajar por construirse una vejez positiva y adaptada que posibilite el descubrimiento de nuevos roles significativos y valiosos para conducirse con mayor seguridad en los contextos vitales. La pasividad se enseñorea y las ganas de vivir descienden en el ser que envejece. La actividad es un excelente medio para abordar la inadaptación, la dependencia y la alienación en un medio social y cultural que la niega a los mayores, a través de sus instituciones, al mismo tiempo que le retira los recursos necesarios para mantenerla. De ahí, que el mantener variados roles interpersonales y sociales se hayan relacionado con elevados niveles de adaptación y satisfacción en edades avanzadas (Bengtson, Rosenthal y Burton, 1990). La vejez es, pues, el resultado de un modo de vivir, pensar y actuar desde que se nace. Se construye desde que se nace.

También desde distintas corrientes de la psicología, como la teoría epigenética de Erikson el enfoque del desarrollo de tareas de Havinghurst..., se ha dedicado especial atención a los procesos de envejecimiento, proponiendo una multiplicidad de paradigmas y visiones del mayor que envejece. Hoy en día parece predominar el enfoque del ciclo vital, quizás debido a su propuesta 


\section{ESTUDIOS Y E N SAYOS}

de combinar lo macro y lo micro; es decir, los procesos sociales y su influencia en la vejez y la dinámica del envejecimiento individual. Su impacto en los proyectos de investigación de carácter longitudinal a través del tiempo ha sido y son reconocidos. Los teóricos del ciclo vital se oponen a las posiciones organicistas por el énfasis que ponen en lo biológico, pero también al determinismo implícito en su descripción del desarrollo, interpretación por la que los mayores -y en realidad todo tipo de personas- están condicionados causal y necesariamente por procesos intrínsecos a su biología y que, consecuentemente, quedan fuera de sus posibilidades.

Frente a ello, tal y como demuestran diversos estudios empíricos, insisten en afirmar que la vejez es una construcción cultural (Bazo, 2004) y el envejecimiento un proceso de naturaleza dinámica y contextual que pone en cuestión cualquier intento de uniformar los comportamientos de los mayores en función de la edad cronológica. A su juicio, los procesos de desarrollo dinámico son de carácter multicausal y multifactorial.

No hay rasgos de personalidad inamovibles ni cambios que puedan pensarse fuera de la interacción de las personas con su entorno sociocultural. Las ideas de proceso y dinámica siguen siendo válidas para el envejecimiento (Baltes y Smith, 2003) y se subraya que durante estos años de desarrollo del ciclo vital se van intercalando, en función de diversos factores contingentes, procesos continuos (acumulativos) y discontinuos (innovadores), en los que se combinan y se compensan ganancias y pérdidas, avances y retrocesos, cuyos fines no siguen un patrón determinado por la edad sino por el nivel de funcionamiento de los mayores cuyo desarrollo psicológico viene caracterizado por la plasticidad intraindividual y la heterogeneidad interindividual (Baltes, Kliegl y Dittmann-Kohli, 1988). En suma, si cabe aceptar que los diferentes tipos de envejecimiento "el normal, que se produce sin enfermedades físicas y mentales, su contrario, el patológico y el óptimo que supone envejecer bajo las mejores condiciones ambientales y personales"(Baltes y Smith, 1990, p. 151) nos permite confirmar que, con el aumento de la edad aumentan, en general, las pérdidas objetivas y subjetivas en la capacidad de reserva, ello no empaña la idea de que el envejecimiento presenta una relevante heterogeneidad. Es decir: en según qué personas, el envejecimiento implica una especificación del proceso general concretándose en cada sujeto en función de sus respectivas particularidades personales y contextuales. O, con otras palabras, tanto la heterogeneidad como la variabilidad interindividual destacan el hecho de que el curso del envejecimiento no tiene por qué derivar en enfermedad y deterioro.

Esta comprensión del desarrollo humano, enriquecido con aportaciones de múltiples ciencias (gerontología, geriatría, genética, biología...) permite entender que los mayores pueden aprender durante toda su vida y que precisamente por el desarrollo alcanzado en distintas fases de sus respectivas vidas cargadas de biografía son más proclives al incremento de la variabilidad interindividual en sus acciones, orientadas, eso sí, por deseos, motivaciones y finalidades personales. Los mayores pueden estar, cuando se sienten motivados para avanzar, en procesos constantes de aprendizaje, lo que supone -aun siendo más difícil para ellos que han nacido y se han desarrollado en entornos escasa o nulamente informáticos- estar abiertos a la adquisición de nuevos saberes y habilidades que les proporcionan las TIC como han confirmado variados estudios (Yuni y Urbano, 2005). 


\section{LAS TIC Y EL ENVEJECIMIENTO ACTIVO}

De todos es sabido que las configuraciones de la actual sociedad tecnológica, de la información o en red, ha modificado la naturaleza y fisonomía de las relaciones económicas (economía financiera global), laborales (sustitución de los sistemas de producción mecánicos por electrónicos, tecnificación de las relaciones de producción, flexibilización de horarios y lugares de trabajo) de la formación (e-learning), del ocio (youtube, redes sociales, chats, juegos online) o comunicacionales (redes sociales, skype, whatssapp, etc.). Todas estas transformaciones nos han llevado a asumir que no se trata solo de un cambio tecnológico, sino de una transformación que afecta a todos los ámbitos de la vida personal y colectiva y que, en consecuencia, lanza importantes retos culturales, políticos, sociales y pedagógicos que los Estados y administraciones competentes no pueden desatender.

En lo específico del tema que nos ocupa, la relación entre las TIC y los mayores, los datos estadísticos ponen de manifiesto dos tendencias que cabe tener en cuenta. Por un lado, se ha dado un enorme crecimiento, al menos en el contexto de los países desarrollados, de las personas mayores que se relacionan y usan las TIC. No obstante, su número y porcentaje sigue siendo notablemente menor al del resto de la población, y muy distanciado del uso que hacen de ellas los jóvenes y adultos. El estudio de 2019 de la Asociación para la Investigación de Medios de Comunicación (AIMC), muestra que frente al 15,7\% de los individuos de entre 25 y 34 años que usan la red, o el 20,7\% de entre 45 y 54 años, sólo el 11,8\% son personas mayores de 65 años. Cuando nos desplazamos desde el uso de los ordenadores al uso de los teléfonos móviles, estas estadísticas cambian significativamente.

La Encuesta sobre Equipamiento y uso de TIC en los hogares (INE, 2019) el 90,7\% de la población de 16 a 74 años ha utilizado Internet, 4,6 puntos más que en 2018. Esto supone un total de 31,7 millones de usuarios, lo que se traduce en un aumento del uso de internet en todos los tramos de edad, aunque el mayor uso de internet sigue siendo una práctica mayoritaria entre los jóvenes de 16 a 24 años 99\%. Con el aumento de la edad desciende el uso de internet, siendo el porcentaje más bajo el que corresponde al grupo de edad de 65 a 74 años (un 63,7\% para los hombres y un $63,5 \%$ para las mujeres). Este estudio también apunta que, cuando se analizan las tipologías de las actividades realizadas por Internet, se observa que tanto en hombres como en mujeres las actividades más realizadas son usar mensajería instantánea, intercambiar mensajes (p. ej. WhastsApp), buscar información sobre bienes y servicios, recibir o enviar correos electrónicos y ver contenidos de video de sitios para compartir (por ejemplo, YouTube).

Es decir, las personas mayores de 65 años muestran mayores dificultades y reticencias que los más jóvenes a la hora de acercarse y utilizar las TIC, pero en la comparativa entre equipamientos tecnológicos, el teléfono móvil es ampliamente más usado que el ordenador. Volveremos más adelante a analizar las causas de estas diferencias.

Los datos acreditados nos hacen caer en una suerte de paradoja que vale la pena explorar. De un lado la evidencia del, todavía, escaso nivel de penetración del uso de las TIC, especialmente en lo que al uso de internet se refiere, entre las personas mayores. Del otro, el hecho de que los mayores suelen contar tanto con una gran disponibilidad de tiempo libre como con algunas necesidades específicas derivadas de su situación social y, en ocasiones, vital. Ambos elementos 


\section{ESTUDIOS Y E N SAYOS}

podrían, en buena medida, ser atendidos si se incrementase el manejo y buen uso de las TIC. ¿Dónde radican pues las causas para que, a pesar de los avances, no acaben de implementarse y explotar estas virtualidades? Según Miranda (2004) dos son los factores principales: 1) la falta de interés y/o el temor hacia lo desconocido de las tecnologías de unos mayores (especialmente los de más de 65 años) que consideran dichas actividades y usos "como cosas de jóvenes"; 2) las limitaciones o barreras propias (nivel de formación, estatus profesional, barreras físicas o motrices, etc.) y del entorno (costes, equipamientos e instalaciones, medio rural). Pensar en las barreras, como limitaciones, de las personas mayores implica necesariamente personalizarlas, de acuerdo a la gran diversidad de potencialidades, necesidades y capacidades que podemos encontrar entre ellos. Pero por ello mismo defendemos que los factores socioeconómicos que generan dificultades o reticencias en su acercamiento a las TIC deben añadirse, y tener especialmente en cuenta, factores de tipo sociocultural y pedagógico. Entre otros motivos porque, como se ha señalado, los mayores no suelen considerar la tecnología como un aprendizaje importante en sí mismo, pero pueden llegar a ver el interés si ella les provee de nuevos medios para otras actividades como, por ejemplo, la comunicación con la familia, los amigos o vecinos de la comunidad (Ala-Mutka et al., 2008).

Acercándonos ahora a los factores que alientan el uso y apropiación de las TIC entre los mayores encontramos un claro interés por las actividades de ocio y entretenimiento (Cefrio, 2011): participación en juegos, chats, comunidades temáticas virtuales, pasatiempos e información general. Diferentes estudios se han hecho eco, también, de la relevancia que tiene en este sentido el empuje, refuerzo y apoyo de los miembros más jóvenes de las familias o de los amigos (Cefrio, 2011, Agudo, Pascual y Fombona, 2012, Fernández y Loredana, 2013). Tras del incremento significativo en el uso de las TIC, y en concreto de los teléfonos móviles-especialmente para llamadas de voz, pero con un progresivo aumento del uso de aplicaciones comunicacionales como WhatsApp y Facebook- encontramos motivos como: a) el interés de los familiares por la seguridad; b) la cercanía y el soporte emocional; c) otras ayudas puntuales como la organización de las rutinas diarias, recordatorios, etc.

Más allá de las motivaciones de ocio, comunicación y relación que acabamos de señalar, podrían aducirse otros motivos y motivaciones que abalarían la importancia y necesidad de introducir las TIC en la vida de las personas mayores. Siguiendo los estudios de Miranda (2004) y Rodríguez (2007) podríamos añadir también razones: 1) terapéuticas: se trata de entornos altamente ricos en estímulos sensoriales que facilitan la activación de funciones cognitivas y sociales, contribuyendo a mantener una mejor calidad de vida en el marco del "envejecimiento activo"; 2) de información y transacciones remotas: interés en tener acceso a informaciones y poder realizar transacciones relevantes para sus situaciones vitales (gestionar pagos de las pensiones y otros movimientos financieros, visitas o controles sanitarios, viajes y actividades, etc.). La accesibilidad a través de Internet beneficia, especialmente, a quienes padecen restricciones de movilidad; 3) formativas. Ligadas a las distintas facetas del aprendizaje a lo largo de toda la vida.

Pero quisiéramos, intentando ir más allá de los simples discursos de buenas intenciones, detenernos a analizar en detalle, a través de algunas investigaciones y proyectos, ciertos factores culturales y pedagógicos que favorecen o entorpecen la motivación por el uso de las TIC por parte de los mayores. 


\section{EST U D I O Y E N SAYOS}

En primer lugar, nos hacemos eco de una investigación realizada por Yuni (1999) centrada en pulsar la opinión de “adultos mayores y ancianos” acerca de la formación que habían recibido en las aulas universitarias a través de los programas pensados para la tercera edad. Se escrutaban en la misma las percepciones de desarrollo y mejora que los mayores tenían de sí mismos, partiendo del supuesto de que "de algún modo están comprometidos en su propio crecimiento y consideran que su proyecto de vida está aún desplegándose” (Yuni, 1999, p. 11). El objetivo, en esta investigación, era considerar si tales efectos contribuyen a prolongar sus vidas en las mejores condiciones físicas, psíquicas y ambientales. Para ello, interrogando a las personas mayores sobre los cambios percibidos por ellos tras la formación recibida (cambios metacognitivos, de autoconcepto, en las relaciones familiares, en el descubrimiento de potencialidades, en la integración social, de hábitos saludables y en la recuperación de actividades pasadas), aparece la actividad como la variable fundamental que atraviesa el movimiento de los mayores y sus respuestas a la formación que recibieron en las Universidades de Tercera Edad (ídem, pp. 345 402). Los datos obtenidos en la investigación ponen de relieve que con la actividad formativas se obtienen no solo conocimientos generales sino también relaciones sociales, desarrollo personal e integración social indicando, a su vez, "que los mayores otorgan a la transmisión de saberes un valor que desborda el objetivo de aumentar su caudal cognitivo, y se vincula a intereses de autonomía, integración, enfrentamiento con sus posibilidades de desarrollo y búsqueda de sentido existencial” (ídem, p. 435). En lo que respecta a la educación, el estudio confirma la "seguridad que la formación da a las personas para enfrentarse a los cambios sociales y tecnológicos”, donde los mayores responden que ella les permite "mostrar menos temor a la tecnología" (ídem, p. 356). No obstante, el autor trata de no magnificar su aportación o convertirla en la panacea de toda educación orientada a la tercera edad.

En segundo lugar, hace una década, tuvimos la oportunidad de participar activamente en un pequeño proyecto de investigación-acción que tenía como finalidad el acercamiento de personas en la tercera etapa de su vida (no vinculadas al mercado de trabajo y que no tienen necesidades formativas vinculadas a él) a las TIC (Gagnon, Riendau y Molina, 2006). El proyecto nació a raíz de la puesta en marcha en Montreal (Canadá) de unos cursos de introducción a la informática llevados a cabo, de manera voluntaria en una asociación de personas mayores, por un doctorando de educación de la UQUàM. Los cursos de iniciación dieron como resultado un deseo explícito y una demanda de ampliación de la formación en herramientas comunicacionales y/o relacionales. Quisimos entonces comprender cómo, por qué y con qué objetivos se acercaban estas personas al conocimiento y uso de las TIC. Organizamos un grupo experimental de personas con un nivel de pericia equivalente que trabajó durante varios meses en el manejo colaborativo de listados de correo electrónicos y herramientas como weblogs, fórum y wiki. Dos hipótesis respecto a la naturaleza de los vínculos y de los modos de aprendizaje quedaban corroboradas tanto por los resultados de nuestro trabajo pedagógico como por las conversaciones mantenidas con los participantes. Ideas que, posteriormente, hemos visto expresadas y verificadas, atendiendo a los necesarios matices, en otros proyectos e investigaciones realizados posteriormente en distintos países (Cefrio, 2011; COTA, 2012; Tatnall, 2014). Por un lado, la naturaleza de los vínculos creados a través del trabajo colaborativo desbordaron pronto los espacios/tiempo de la actividad. Se fraguaron nuevas relaciones de amistad que, para algunos de los participantes, especialmente los casos de varias personas de entre 75 y 80 años que vivían solas, fueron importantes como modo 


\section{ESTUDIOS Y E N SAYOS}

de romper con cierta soledad y aislamiento social. La motivación por los aprendizajes se dirigió, rápidamente, a la comunicación con familiares, amigos y seres queridos distantes. Algunas de las personas tenían hijos viviendo en Europa o Norteamérica, por lo que el uso de las tecnologías para poder comunicar, tanto con ellos (correo electrónico o el por entonces incipiente Skype) como con los amigos en el duro invierno canadiense, apareció como demanda y motivación desde el comienzo. Por otro lado, nuestro trabajo pedagógico y las observaciones cotidianas nos permitieron percibir e ir poniendo en práctica modos de acercamiento, orientación y enseñanza más afinados y efectivos a la hora de traspasar los temores de los mayores frente al universo tecnológico. Se convirtió en una cuestión de primera relevancia generar un entorno de aprendizaje amigable y seguro, así como asegurar un acompañamiento continuado e individualizado -en el aula, al teléfono, online y, en ocasiones y con las personas de mayor edad y dificultad, en sus domicilios-para el ritmo y progreso de cada grupo y persona en su proceso de familiarización con los nuevos hardware y software utilizados.

En definitiva, la presencia y el empuje de los cercanos (amigos, familia y formadores), así como los deseos de comunicar con ellos, se mostraron como elementos cruciales en un proceso de adquisición de competencias y usos tecnológicos.

\section{LAS TIC Y LAS PROMESAS HECHAS A LOS MAYORES}

Hace unos años, Abad (2014) acertaba al señalar que el proceso de acercamiento y de alfabetización tecnológica de los mayores requeriría de tres fases. En primer lugar, políticas decididas que se esfuercen en disminuir la brecha digital generacional mediante la dotación de medios e infraestructuras de red que permitan el acceso de los mayores. En segundo, una formación adecuada en alfabetización y competencias digitales que aliente el uso de los medios dispuestos. La tercera fase podría centrarse en el desarrollo, promoción y difusión de productos y servicios (comercio, administración, e-learning, ocio, relaciones personales, etc.). Lo que nos interesa resaltar del análisis de Abad (2014), al que nos adherimos en buena medida, es que ubica la posibilidad de éxito de las iniciativas en un camino que atraviesa tres momentos en los que confluyen y se combinan responsabilidades políticas, sociales y pedagógicas. Su propuesta indica el recorrido de los retos que pueden alumbrar nuestra pregunta de partida.

\subsection{Retos en lo político}

No es difícil deducir de lo desarrollado hasta ahora que hay distintas formas de interpretar en España la noción de envejecimiento activo. Su filosofía, en general, promueve al tratar de aplicarla contradicciones en determinadas geografías y en contextos más locales y comunitarios dónde se visualizan mejor los efectos de estas políticas. Por ejemplo, apuestan por aumentar la esperanza de vida, pero también son más numerosos los casos de muertes prematuras o, por poner otro ejemplo, defienden en sus pórticos introductorios la mejora de la calidad de vida y, al mismo tiempo, se enfatiza la medicalización del envejecimiento centrándose en las patologías y enfermedades. La otra forma de pensar y de recrear las políticas y programas que nos propone el envejecimiento activo no parece estar dando el resultado esperado (Sánchez y Ferreiro, 2010). Tales efectos resaltan la crítica de que predomina más la retórica de los discursos antes que la 


\section{ESTUDIOS Y E N SAYOS}

materialización de sus propuestas. Y es que está por clarificar cómo se va superar la paradoja que vive Europa dominada por las lógicas neoliberales y mercantiles -en dónde se ha producido una gran fragmentación social y un individualismo preocupante-que desvinculan a los mayores del resto social-a su vez, individualizado- mientras declara propugnar políticas en favor de los mayores (Sáez, Sánchez y Pinazo, 2008). Pero estas se han mostrado tan dispersas y sectoriales como inconsistentes, sobre todo si se observa que aquellas orientadas a la protección acaban fijándose exclusivamente en una cuestión de empleo, o aquellas políticas pensadas para la jubilación finalizan escorándose a solo programas de prevención de la salud con los débiles resultados hoy conocidos (Bazo, 2004). Se precisa un enfoque político que supere planteamientos excesivamente tecnocráticos y nos permita debatir socialmente la finalidad que deseamos satisfacer con las TIC y qué funciones queremos que cumplan (Brunner y Tedesco, 2003). Con todo, en actividades aisladas, es preciso reconocer que el espíritu del envejecimiento ha movido a las comunidades y localidades que, en relación con las TIC, han impulsado programas, prácticas y experiencias bajo el auspicio de políticas vinculadas al bienestar, la convivencia, la participación, la seguridad y protección de los mayores, la educación (así, son conocidos los Programas de Atención Jurídica, el teléfono de Atención Diaria, la Teleasistencia, Universidades de la Tercera Edad, etc. ...) en diferentes contextos españoles.

Dos importantes documentos nos permiten profundizar en la cuestión que nos ocupa. En primer lugar, la Declaración de principios y los compromisos derivados de la Cumbre Mundial sobre la Sociedad de la Información (CMSI, 2004), celebrada en Ginebra y Túnez entre 2003 y 2005, entidad dependiente de la ONU. En segundo lugar, nos hacemos eco del estudio de la Comisión Europea sobre Envejecimiento Activo y potencial de las TIC para el aprendizaje (AlaMutka et al., 2008). En lo que respecta al primer documento nos detenemos, entre otras medidas, en la instancia a los gobiernos a que trabajen en dos líneas de actuación:

1. Implementar condiciones de conectividad y acceso universal a la infraestructura y servicios TIC. La CMSI reconoce que las TIC son un medio y no un fin en sí mismas; un medio que en condiciones favorables pueden ser un instrumento eficaz para acrecentar la productividad, el crecimiento económico, el empleo; para promover el diálogo entre las personas y las naciones; para mejorar, en definitiva, la calidad de vida de todos.

2. El fomento de un clima de confianza y seguridad, de la información y la de las redes. Para que todo ello acaezca debe formarse a los mayores de modo tal que puedan entender los beneficios que las TIC encierran para ellos. La tecnología no es neutral ni un instrumento perverso, es un medio de comunicación de diverso rango de aplicación, ya que no todas estas herramientas ofertan similares capacidades y se diferencian tanto por el abanico de sus realizaciones como por las limitaciones y competencias de cada una de ellas.

Es además cuestionable sacar como conclusión que nuestra sociedad se va a volver más justa, libre o democrática simplemente por el uso de estos nuevos medios. Es la vieja utopía de la ciudad interconectada y cableada dónde todo el mundo habla con todo el mundo. A lo sumo, lo tecnológico puede hacer la democracia más eficiente pero no provee, por sí sola, de unas estructuras democráticas totales, tal como apuntaba Sádaba (2002). 


\section{ESTUDIOS Y E N SAYOS}

Sin embargo, si se revisa detenidamente, el impacto de las TIC en los procesos sociales es, en algunos casos, evidente. Internet, por ejemplo, no es solo un instrumento, sino un medo de comunicación poderoso, ello hasta el punto de que ciertos problemas sociales han perdido visibilidad o están siendo replanteados de otra manera. No existe, es cierto, una solución técnica a problemas políticos y sociales, pero ellas pueden abrir o ampliar brechas de desigualdad como también contribuir a promover asociaciones y redes de base. Esta reconversión mental, este recrear pedagógico que demanda la adaptación a las nuevas tecnologías viene condicionado, en el caso de los mayores envejeciendo, por ciertas rupturas generacionales que les enfrenta a problemas inesperados. En suma, las políticas que se implementen en favor del acceso y uso de las TIC, aun reconociendo que siempre serán bienvenidas, deben enmarcarse dentro de un amplio paraguas de políticas, en buena medida, contra la pobreza, la desigualdad, la exclusión y la vulnerabilidad. Situaciones agravadas por los efectos del neoliberalismo en nuestras vidas, debido al impacto que las condiciones socioeconómicas tienen sobre las posibilidades de envejecer bien y el papel que pueden cumplir los usos de las tecnologías en estos procesos vitales.

\subsection{Retos en lo pedagógico}

La incorporación de las TIC a la educación es, pues, una cuestión de estrategia global de política social y educativa. ¿Temas a tener en cuenta? Aspectos tan importantes como: 1. Que los consensos entre los ministerios públicos de educación con las agencias -universidades, centros de secundaria, áreas de comunicación y formación en las comunidades...- públicas y privadas permitan ir más allá de las lógicas de mercado imperantes en esto de la tecnología. 2. Que desde esta posición institucional pública se estudie y se informe sobre el valor real -no solo potencialde lo que supone incorporar las TIC a las necesidades, sociales, personales y educativas, de las personas mayores y, por ende, se termine con el juego de promesas que en diferentes experiencias se ponen en juego con ánimo interesado mercantil. 3. Que se tenga presente a educadores y formadores ya que la utilización de las TIC modifica algunas de sus funciones tradicionales en los procesos de aprendizaje, tanto dentro del sistema educativo formal como en el no-formal. 4 . Que se evalúen periódicamente las experiencias e innovaciones que con las nuevas tecnologías de la información y comunicación se lleven a cabo (Brunner y Tedesco, 2003, pp. 8-9) a fin de identificar los mejores trayectos que faciliten el acceso universal a ellas y eviten marginaciones de toda índole, cosa que acontece con las personas mayores.

Lograr aumentar el interés de los mayores por las TIC, así como incrementar su alfabetización y su uso digital, requeriría de dos tipos de trabajo eminentemente pedagógico. Por un lado, es preciso seguir trabajando desde amplias campañas de información y difusión, pero también desde una formación de cercanía, que combatan las barreras y temores culturales y generacionales. Por otro, hay que repensar y mejorar la gama de programas formativos, recogiendo algunas buenas prácticas, pero superando la situación de experimentación y bricolaje habitual de los mismos, para lograr adaptarlos a las características de una población heterogénea y diversa en sus necesidades y deseos. Resulta evidente que, entre los mayores de hoy en día, en lo que se refiere al uso y manejo de TIC, podemos encontrarnos con niveles que variarían entre los que deben introducirse todavía en el primer suelo de la alfabetización digital, la competencia digital; los que buscan mejorar 


\section{ESTUDIOS Y E N SAYOS}

y ampliar su uso digital y los que aspiran a una transformación digital que estimule cambios significativos en sus conocimientos y en sus contextos personales y sociales (Abad, 2014).

En el primer tipo de trabajo, y siempre hablando de aquellos mayores que no han trabajado en contextos en los que se hacía necesario aplicar conocimientos tecnológicos, hay que tener en cuenta que la primera barrera es la propia resistencia al cambio. El aprendizaje de nuevos lenguajes y códigos no resulta sencillo para nadie. Pero si a esta "resistencia natural” añadimos que tales aprendizajes se desarrollan en un contexto o medio también desconocido y a primera vista hostil, los temores aumentan exponencialmente. Bajo la falta de interés y/o la excusa de la edad se esconde, en demasiadas ocasiones, una falta de seguridad respecto al uso de las infraestructuras y de sus propias capacidades para enfrentar las tareas. La oferta de formas cercanas de acompañamiento y la adhesión a métodos pedagógicos diferenciados de los utilizados con poblaciones más jóvenes y socializadas en contextos de alta presencia de TIC, se han revelado como estrategias exitosas a la hora de destrabar estos frenos. En lo relativo a las formas de acompañamiento cabría prestar atención no solo a la formación por parte de expertos, sino también a la posibilidad de realizar acompañamientos presenciales y a distancia en cuestiones relativas a incidencias técnicas que impiden realizar actividades, así como tareas de asesoramiento continuado (coaching) por parte de personas cercanas (Cefrio, 2011). Las universidades de Tercera Edad, también llamadas de la experiencia, han dado importantes pasos al utilizar y dedicar módulos y cursos especializados sobre la utilización del ordenador.

Por último, la posibilidad de adaptar los métodos pedagógicos, como se desprende de las investigaciones citadas, requiere de un trabajo previo que aborde no solo las acostumbradas tipologías de uso de internet, sinolos aspectos (sociales, culturales y personales) que condicionan su uso por parte de los mayores. Por lo mismo Abad (2014) reclama, y nosotros con él, que los programas de alfabetización digital se diseñen de acuerdo a principios de contextualismo (ajustar los materiales al entorno cultural y social, diferenciando a las personas en función de sus dependencias, lazos sociales, intereses, etc.); incrementalismo (decidir el desarrollo de cada fase en función de niveles de competencias); y motivación (que posibilita evaluar la receptividad de los procedimientos y 1 proceso de absorción señalando las mejores formas de acceso a los conocimientos y habilidades en función de la valoración de su empleo exitoso).

\section{A MODO DE REFLEXIÓN ABIERTA: ¿SE HA CUMPLIDO LA PROMESA?}

Es tiempo de responder breve y concisamente a la pregunta que daba título a nuestro trabajo, articulándolo. Aunque se puede decir que, en general, las personas mayores son reticentes a usar las nuevas tecnologías, como se sostiene en el trabajo de Pérez (2015), en el que un 14,7\% de los encuestados manifiesta que está poco dispuesto a utilizar las TIC, aunque se aclara que este hecho se reduce con la realización de actividades culturales. Así, acorde con lo argumentado, puede afirmarse que las políticas y las pedagogías que intentan acercar las personas mayores a las TIC para mejorar sus condiciones de vida y favorecer el envejecimiento activo, solo cumplirán sus promesas si, más allá de declaraciones de intenciones o alabanzas generalistas, son capaces de percibir las formas óptimas en que los mayores pueden apropiarse y aprovecharse de ellas para tareas, objetivos y finalidades directamente percibidos y sentidos por ellos. Es importante partir de sus 


\section{EST U D O S Y E S A Y S}

deseos de los mayores, de sus necesidades como punto de partida en la relación entre las personas mayores y las TIC. Pensamos que, a lo largo de estas páginas, hemos dado cumplida cuenta de la relevancia que factores comunicativos, relacionales y afectivos juegan en este proceso.

\section{REFERENCIAS}

Abad, L. (2014). Diseño de programas de e-inclusión para alfabetización mediática de personas mayores. Comunicar, 42(XXI), 173-180. doi: 10.3916/C42-2014-17

Agudo, S., Pascual, M.A. y Fombona, J. (2012). Usos de las herramientas digitales entre las personas mayores. Comunicar, 39 (XX), 193-201. doi: 10.3916/C39-2012-03-10

Ala-Mutka, K., Malanowski, N., Punie, \& Cabrera, M. (2008). Active Ageing and the Potential of ICT for Learning. IPTS \& EuropeanCommission. Recuperado de http://ftp.jrc.es

Arendt, H. (1993). La condición humana. Barcelona: Paidós.

Asociación para la Investigación de Medios de Comunicación (2019). Audiencia de Internet. Perfil por edad de los usuarios. Recuperado de http://internet.aimc.es

Baltes, P.B.y Smith, J. (2003). New frontiers in the future of aging: from successful aging of the young old to the dilemmas of the fourth age. Gerontology, 49, 123-135.

Baltes, P.B.; Kliegel, R. \&Dittmann, F. (1988). On the locus of training in research on the plasticity of fluid intelligence in old age. Journal Educational Psychology, 80, 392-400.

Bazo, M.T. (2004). Envejecimiento y familia. Arbor: Ciencia, pensamiento y cultura, 702, 323-344.

Benjamin, A.E. y Estes, C. (2003). Intervenciones sociales con mayores. En J. Sáez (Coord.), Educación y aprendizaje en las personas mayores (pp. 79-100). Madrid: Dykinson.

Bengtson, V.L., Rosenthal,C y Burton, L.(1990). Families and Aging: Diversity and heterogeneity. En R.H.Binstock y L.K.George, (Edits.), Handbook of Aging and the Social Sciences (pp. 263-287). San Diego: Academic Press.

Brunner, J.J. y Tedesco, J.C. (Edits.) (2003). Las nuevas tecnologías y el futuro de la educación. B. Aires: Septiembre Grupo Editor

Caro, F. y Sánchez, M. (2005). Envejecimiento productivo. Concepto y factores explicativos. En S. Pinazo y M. Sánchez (Dir.), Gerontología. Actualización, innovación y propuestas (pp. 457-490). Madrid: Pearson.

Cefrio (2011). Génération @. Portrait de l'utilisation d'internet et de l'ordinateur par les aînés internautes du Québec. Recuperado de http://www.cefrio.qc.ca/

CMSI (2003). Declaración de principios. Construir la Sociedad de la Información: un desafío para el nuevo milenio. Recuperado de https://www.itu.int/

COTA (2012). Older Victorians online report. Recuperado de http://cotavic.org.au/

Fernández-Ardèvol, M. \&Loredana, I. (2013). Older people and mobile communication in two European contexts. Romanian Journal of Communication and Public Relations, 15(3), 83-98. Recuperado de http://journalofcommunication.ro/ 


\section{EST U D O S Y E SAYOS}

Fernández- Ballesteros, R. (2009). Envejecimiento activo: contribuciones de la psicología. Madrid: Pirámide.

Fusaro, M. (Dir.) (2007). Le rôle des ainées dansle transfert intergénérationnel des technologies del'information et de la communication au sein de la cellule familiale. Québec: CRSH. Recuperado de http://www. intertic.uqam

Gagnon, M., Riendau, J. y García Molina, J. (2006). Entornos informáticos en Educación Social: una propuesta de investigación. Revista de Pedagogía Social, (12-13), 181-191.

INE (2019). Población que usa Internet (en los últimos tres meses). Tipo de actividades realizadas por Internet. Recuperado de https://www.ine.es/

Miranda de Larra, R. (2004). Los Mayores en la Sociedad de la Información: situación actualy retos de futuro. Madrid: Fundación Auna. Recuperado de http://www.financialtech-mag.com/

OMS (2002). Envejecimiento Activo: un marco político. Madrid: Asamblea Mundial sobre Envejecimiento.

Pavón, F. (2000). Tecnologías avanzadas: nuevos retos de comunicación para los mayores. Comunicar, $15,133-139$.

Pérez Muñoz, M. (2015). La educación a través del arte y las relaciones intergeneracionales: el ciney las personas mayores (tesis doctoral). Universidad de Murcia. Departamento de Teoría e Historia de la Educación, Facultad de Educación.

Rodríguez, R. M. (2007). Personas mayores y aprendizaje a lo largo de la vida de las tecnologías de la información y la comunicación. Pixel-Bit. Revista de Medios y Educación, 30, 41-48.

Sádaba, I. (2002). Nuevas tecnologías y política: acción colectiva y movimientos sociales en la sociedad de la información. Recuperado de http://www.uned.es

Sáez, J., Pinazo, S. y Sánchez, M. (2008). La construcción de los conceptos y su uso en las políticas sociales orientadas a la vejez: la noción de exclusión y vulnerabilidad en el marco del envejecimiento, Revista del Ministerio de Trabajo e Inmigración, 75, 75-94.

Sáez, J. (2010). La educación de personas mayores: de la lógica disciplinar a la lógica profesional. En A. Cabedo (Edit.), La educación permanente: la universidad y las personas mayores (pp. 263-303). Castellón de la Plana: Publicacions de la Universitat Jaume I.

Sánchez, M. y Ferreiro, J. (2010). Las promesas del envejecimiento activo: investigación, desarrollo e innovación en Europa. El Escorial/Madrid: Informe/Resumen.

Tatnall, A. (2014). ICT, education and older people in Australia: A socio-technical analysis. Education and Information Technologies, 19(3), 549-564.

Wong, Y. Ch; Chen, H.; Lee, V.; Fung, J. \& Law, Ch. (2013). Empowerment of Senior Citizens via the Learning of Information and Communication Technology. Agein International, 39(2), 144-162.

Yuni, J.A. (1999). Optimización del desarrollo personal mediante la intervención educativa en la adultezy en la vejez. Granada: Universidad de Granada.

Yuni, J.A. y Urbano, C. (2005). Educación de Adultos Mayores: teorías, investigaciones e intervenciones. Córdoba: Edit. Brujas. 